Amman, Jordan (Al-Qudah AA et al. Pediatr Neurol 1990; 6:57-59). Four $\mathrm{DMD}$ patients were studied prospectively by cranial $\mathrm{MRI}$, DNA deletion analysis, clinical evaluation and IQ testing. There was no significant correlation between verbal IQ and MRI findings, DNA deletion or the clinical severity of the disease. Other than mild atrophy in two patients, no significant anatamical brain lesion was discovered. Previous reports have attributed intellectual impairment in $\mathrm{MM}$ to anatomic brain changes, abnormal dendritic development, and migrational lesions. Others have described a direct relation between the severity of clinical disease and impairment of IQ. (Rosman NP Brain 1966 $89: 769)$.

\title{
GENETICS OF DUCHENNE AND BECKER DYSTROPHIES
}

The molecular basis for Duchenne and Becker muscular dystrophies is reviewed from the Department of Pediatric Neurology, Floating Hospital for Infants and Children, New England Medical Center Hospitals and Tufts University, Boston, Mass. Until recently the diagnosis of $\mathrm{DMD}$ or BMD depended on clinical signs and symptoms, serum creatine kinase, EMG and muscle biopsy. The isolation of the gene defective in $\mathrm{DMD}$ and $\mathrm{BMD}$ and the identification of dystrophin have revolutionized the diagnostic issues. The mutated gene causing Duchenne and its allelic milder Becker phenotype has been assigned to band P21 of the short arm of the $\mathrm{X}$ chromosame (Xp21). Dystrophin has been characterized by DNA sequencing and by immunologic studies. When the family history is negative for DMD and BMD the Western Blot assay of protein derived from a specimen can confirm the clinical diagnosis of DMD or BMD and can be used to predict the severity of the disease. If the dystrophin assay result is abnormal DNA analysis should be performed. Detection of a dystrophin gene deletion will facilitate carrier detection and prenatal diagnosis in the proband's family. If no deletion or duplication is found, linkage analysis may be attempted for prenatal diagnosis and carrier detection. Peripheral blood DNA may be used for Southern Blot testing if a muscle biopsy specimen is not available and may confirm the diagnosis in more than $65 \%$ of the cases. In typical cases of IND or BMD with a family history of $\mathrm{X}$ linked muscular dystrophy, linkage analysis is unnecessary if the clinical diagnosis has been confirmed in an affected family member by analysis of dystrophin or DNA or both. The less invasive polymerase chain reaction test may be used if the diagnosis has not been confirmed by dystrophin or DNA analysis in other family members. Muscle biopsy for dystrophin analysis will be required in families without a clear cut $X$ linked pattern of inheritance or in families with both male and female siblings affected, suggesting an autosomal recessive form of $M$ (Darras BT. Molecular Genetics of Duchenne and Becker muscular dystrophy. J Pediat July $1990 ; 117: 1-15)$.

COMMENT: Gross abnormalities of the dystrophin gene may still result in a partially functional dystrophin protein and a relatively mild clinical progression, compatible with a diagnosis of Becker muscular dystrophy. Angelini C et a. (Neurology May 
$1990 ; 40: 808)$ describe a patient with a duplication of more than $400,000 \mathrm{bp}$ of the dystrophin gene, the largest characterized to date. The propositus a 13 year old boy presented at age 4 with myalgia and cramps after exercise or running in the cold. The CK ranged from 1400 to $8630 \mathrm{U} / 1$, the EMG showed small polyphasic motor units, and the muscle biopsy revealed a mild myopathic picture with scattered atrophic and hypertrophic muscle fibers, a few degenerating fibers and a mild inflammatory reaction. Electronmicroscopy showed hypertrophic ring fibers. Leg muscle ultrasound revealed scattered fibrosis. He was treated for five months with prednisone at $50 \mathrm{mg} / \mathrm{d}$ followed by two months at $\mathbf{5 0}$ mg on alternating days. The $\mathbf{C K}$ levels declined and the child had less muscle pain.

\section{LEARNING AND BEHAVIOR DISORDERS}

\section{STIMULANT MEDICATION FOR ADHD}

The use of stimulant medication by primary care physicians in treatment of attention deficit hyperactivity disorder was evaluated by a random national survey of family practitioners and direct screening of 457 patients in midwestern cities and is reported from the Division of Developmental Disabilities, Departments of Pediatrics and Preventive Medicine University of Iowa, Iowa City and Department of Psychology, University of Kentucky, Lexington. In the national survey, methylphenidate was used by $85 \%$ of pediatricians, dextroamphetamine $11 \%$, and pemoline $23 \%$. Pediatricians used stimulant medication in preschool children and adolescents as well as children between 6 and 16 years of age. Drug holidays were employed by more than $2 / 3$ rds of physicians, but placebo trials were rarely used. Height and weight were monitored by three-quarters of the physicians. Timed or sustained release forms of methylphenidate were used by less than $50 \%$. The Feingold diet and sugar restricted diets were employed by 10 and $15 \%$, respectively, and behavior modification by $77 \%$. In the direct patient screening, the prevalence of $\mathrm{ADHD}$ diagnosis was $5.3 \%$ of all elementary school aged children screened, and $88 \%$ were treated with methylphenidate. Medication was considered effective by the parents of $85 \%$ of children and efficacy was unrelated to the accuracy of the diagnosis. When DSM-III-R criteria were used, only $72 \%$ of those assigned a diagnosis of $\mathrm{ADHD}$ by physicians would qualify on a psychiatric interview of the parents and only 53\% on a teacher report of symptoms. Although physicians reported the use of behavioral treatments, parents reported infrequent use of behavior modification. (Wolraich $\mathrm{ML}$ et al. Stimulant medication use by primary care physicians in the treatment of attention deficit hyperactivity disorder.

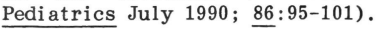

COMENT: The authors concluded that systematic behavioral treatments were underused compared to medications and the accurate diagnosis of ADHD needs clarification by identification of subtypes. 